Aus der Inneren Abteilung des Krankenhauses der Jüdischen Gemeinde in Berlin.

(Dirigierender Arzt: Prof. Dr. J. Lazarus.)

\section{Die Aussichten der Sauerstoff-Inhalationen nach den neuesten physiologischen Unter- suchungen.}

\author{
Von Dr. E. Aron, Assistenzarzt.
}

Wir haben nach sehr interessanten und neuen Versuchen von Zuntz und Loewy ${ }^{1}$ ) erfahren, daß unsere bisherigen Anschauungen über den normalen Sättigungsgrad des arteriellen Blutes mit Sauerstoff keine ganz zutreffenden gewesen sind. Es hat sich ergeben, dal das arterielle Blut nicht $\mathrm{zu}^{14} / 15$, beziehungsweise $9 / 10$, sondern nur zu $82 \%$ mit Sauerstoff gesättigt ist. Bei dieser neuen Sachlage, welche wir als richtig akzeptieren wollen, könnten sich möglicherweise sehr wesentliche Wandlungen unserer Anschauungen über die Möglichkeit einer stärkeren und bedeutungsvollen Sauerstoffaufnahme des Blutes bei Einatmung reinen Sauerstoffs ergeben. Um zu entscheiden, ob dies wirklich zutrifft, werden wir eine Berechnung darüber anstellen müssen, wie groß die Sauerstoffaufnahme des Blutes bei Einatmung reinen Sauerstoffs sein würde bei Zugrundelegung dieser neuen Werte. Wir wollen bej dieser sehr einfachen Berechnung von der denkbar günstigsten Möglichkeit ausgehen, daß es wirklich zutrifft, bei Einatmung von reinem Sauerstoff das Blut mit Sauerstoff $\mathrm{zu}$ sättigen. $0 \mathrm{~b}$ jedoch eine solche volle Sauerstoffsättigung des Blutes bei der zu therapeutischen Zwecken üblichen Sauerstoff-Applikation je eintreten kann, das werden wir später zu erörtern haben und danach die notwendigen Korrekturen an den Zahlen, welche wir erhalten werden, anbringen müssen.

Bei unserer Berechnung wollen wir zunächst feststellen, wie viel Sauerstoff ein normaler Mensch bei Einatmung von reinem Sauerstoff aufnehmen würde, vorausgesetzt, daß sich dabei das Blut mit Sauerstoff sättigte. Wir wissen, daß ein gesunder Mensch im Durchschnitt 8-900 g Sauerstoff in 24 Stunden aufnimmt. Bei voller Sauerstoffsättigung des Blutes würde ein Mensch $18 \%$ Sauerstoff mehr aufnehmen können. Es würde sich daher eine Mehraufnahme von $9 \times 18=162$ g Sauerstoff ergeben. Dazu muißte freilich volle 24 Stunden reiner Sauerstoff eingeatmet werden. Bei unseren therapeutischen SauerstoffInhalationen lassen wir jedoch nur relativ kurze Zeit Sauerstoff inspirieren, meist wohl nicht einmal eine volle Stunde innerhalb 24 Stunden. Jene $162 \mathrm{~g}$ Sauerstoff wären also durch $24 \mathrm{zu}$ dividieren. Wir w ürden demnach in dem denkbargünstigsten Falle eine Mehraufnahme von 6,75 Sauerstoff bei einem normalen Menschen erhalten. Daß eine etwaige Mehraufnahme von Sauerstoff für den gesunden Menschen gar keine Bedeutung hat und als etwas Ueberflüssiges vom Tierkörper wieder ausgeschieden wird, darüber sind wir uns wohl einig. Es ist dies durch die exakten, neuen Untersuchungen von $\mathrm{Zuntz}$ und Durig ${ }^{2}$ ) von neuem bestätigt worden. Diese Untersuchungen waren notwendig und zeitgemäß, da die Rosenthalschen Experimente ${ }^{3}$ ) danach angetan waren, unsere Anschauungen in dieser Hinsicht eventuell zu verwirren.

Nachdem wir bisher beim gesunden Menschen die einschlägigen Werte kennen gelernt haben, wollen wir nunmehr eine ähnliche Berechnung beim kranken Menschen anstellen. Wir werden selbstredend solche krankhaften Zustände ins Auge fassen müssen, bei denen die Sauerstofftherapie besonders empfohlen wird. Da begegnet man aber sofort recht großen Schwierigkeiten. Es ist sehr mißlich, sich ein geeignetes Krankheitsbild herauszugreifen, da die Autoren, welche die Sauerstofftherapie ganz besonders warm empfehlen, sich hier ganz merkwürdig widersprechen. Die Einen behaupten, überraschende Erfolge von der Sauerstofftherapie gesehen zu haben bei Krankheiten, bei denen die An-

1) Ueber den Mechanismus der Sauerstoffversorgung des Körpers. Arahiv f. Anat. 1. Phys. Phys. Abt. 1904, S. 166. - 2) A. Durig, Ueber Aufnahme und Verbrauch von u. Phys. Phys. Abt. 1904, S. 166. - 2) A. Durig, Ueber Aufnahme und Verbrauch von
Sauerstoff bei Aenderung seines Partiardruckes in der Alveolenluft. Arch. f. Anat. u. Sauerstoff bei Aenderung seines Partiardruckes in der Alveolenluft. Arch. f. Anat. u.
Phys., Phys. Abt. Euppl. Bd. 1903, S. 209. N. Zuntz, Physiolog. Ges., Sitzung vom 24. April Phys., Phys. Abt. Suppl. Bd. 1903, S. 209. N. Zunt 2, Physiolog. Ges., Sitzung vom 24. April
1903 , S. 492. - 3) Rose nthal, Untersuchungen über den respiratorischen Stoffwechsel. Arch. f. Anat. u. Phys., Phys. Abt. 1902, Heft 3 u. 4. deren einen Erfolg für unmöglich halten, und umgekehrt. Diese gewib sehr interessante Tatsache dürfte dazu dienen, die Unsicherheit, um nicht zu sagen Unwirksamkeit der Sauerstofftherapie zu erweisen. Nun soll die Sauerstofftherapie ganz besonders zweckmäßig und erfolgreich bei acuten Anämien infolge von stärkeren Blutverlusten sein. Es dürfte daher vielleicht indiziert sein, diese Berechnung zunächst bei einem stärkeren Blutverluste anzustellen. Wir wollen annehmen, daß bei der Blutung beträchtliche Mengen des Gesamtblutes, wobei eine natürliche Kompensation nicht mehr möglich ist, verloren gegangen seien. Es ist dabei eine sichere Cyanose und Dyspnoë zustande gekommen. Nehmen wir an, daß so viel $\left.{ }^{1}\right)$ Blut verloren gegangen sei, daß der anämische Körper ein Viertel Sauerstoff weniger aufnehmen kann als unter normalen Verhältnissen, statt 900 also nur $900 \_900 / 4=900-225$ $=675 \mathrm{~g}$ Sauerstoff in 24 Stunden bei Einatmung gewöhnlicher Atmosphärenluft. Installieren wir nun Sauerstoff-Inhalationen, so kann sich nach unseren heutigen Kenntnissen im allergünstigsten Falle die Sauerstoffaufnahme um $18 \%$ steigern, wenn eine volle Sauerstoffsättigung des Blutes zustande käme. Es würden dann von dem blutarmen Organismus $675+121,5=796,5 \mathrm{~g}$ Sauerstoff aufgenommen werden, wenn diese Sauerstoffatmung und Sauerstoffsättigung des Blutes 24 Stunden lang unterhalten werden könnte. In einer Stunde würde die Mehraufnahme des Körpers an Sauerstoff nur 121,5:24=5,06 Sauerstoff betragen. Statt 675 würde der blutarme Mensch also 680,06 Sauerstoff aufnehmen, wenn er eine volle Stunde reinen Sauerstoff einatmete und auf diese Weise sein Blut mit Sauerstoff sättigen könnte. Diese Mehraufnahme von 5,06 Sauerstoff muß man sich auf 24 Stunden verteilt denken. Es läßt sich weiter leicht berechnen, eine wie große Mehraufnahme an Sauerstoff ungefähr auf den einzelnen Atemzug entfallen würde. Praeter propter würden nicht volle $5,62 \mathrm{ccm}$ Sauerstoff pro Atmung vom kranken Körper mehr aufgenommen werden. (In einem einzigen Atemzuge werden zirka $40 \mathrm{ccm}$ Sauerstoff von einem normalen Menschen aufgenommen.) Hierzu käme noch eine Steigerung des im Blute einfach absorbierten Sanerstoffs um das Fünffache, von $0,5-0,6 \%$ Sauerstoff auf $2-3 \%$ Sauerstoff. Eine genaue Berechnung dieses Wertes ist wohl nicht durchführbar, da wir ja nicht genau wissen, wie viel von den 900 g Sauerstoff, welche der Mensch in 24 Stunden aufnimmt, vom Hämoglobin, und wie viel als im Blute einfach absorbierter Sauerstoff zu den Zellen des Körpers transportiert wird. Jedenfalls müssen wir beachten, daß eine erhebliche Menge Blut verloren gegangen ist. Wenn wir also die oben abgeleiteten Zahlen berücksichtigen, so scheinen mir persönlich dieselben keineswegs so grop zu sein, daß ich bei dieser Sachlage mit einer derartigen Mehraufnahme an Sauerstoff eine Lebensrettung motivieren möchte. Ganz besonders wird dies dann in die Augen springen, wenn wir an jenen Zahlen noch weitere Reduktionen anbringen müssen. Eine volle Sauerstoffsättigung des Blutes ist, wie wir sehen werden, bei der sehr unvollkommenen Sauerstoff-Inhalationsmethode der Praxis nicht nur unerwiesen, sondern unmöglich.

Handelt es sich statt um akute Blutverluste um andere Bluterkrankungen, wie schwere Chlorosen, Anämien, Leukämien, so können wir die oben abgeleiteten Zahlen fast ohne weiteres verwerten, da es für die Sauerstoffaufnahme des Blutes ziemlich gleichgültig ist, ob Blut verloren gegangen, oder ob nur die Menge des sauerstoffbindenden Hämoglobins verringert ist. Für den einfach absorbierten Sauerstoff liegen die Verhältnisse etwas anders, da bei diesen Erkrankungen die Gesamtmenge des Blutes nicht verringert sein muß.

Wollen wir eine ähnliche Berechnung der Sauerstoffaufnahme für andere einschlägige pathologische Prozesse ausführen, so hat das gar keine Schwierigkeiten. Käme bei Stenosen der luftzuführenden Atemwege z. B. 1/4 Luft weniger in die Lunge als unter normalen Verhältnissen, so würde das Blut bei Einatmung von gewöhnlicher Luft auch $1 / 4$ weniger Sauerstoff aufnehmen. Bei Einatmung von reinem Sauerstoff könnte die aufgenommene Sauerstoffmenge um 12\% steigen, falls das Blut sich dabei mit Sauerstoff sättigte. Es würden sich also für Stenosen der Luftwege die gleichen Zahlen ergeben, welche wir früher bei Besprechung acuter Blutverluste entwickelt haben.

1) Wir haben absichtlich einen größeren Blutverlust angenommen, da die Saurerstofftherapie ja dann einsetzen soll, wenn natürliche Kompensationen nicht ausreichen. 
Für Pneumonien, Pleuritiden, Pneumothorax, wobei infolge der Krankheit Teile der Lunge von der Atmung ausgeschaltet sind, bei Herzfehlern, bei denen infolge Verlangsamung der Blutzirkulation die Cyanose bedingt wird, lassen sich die oben abgeleiteten Werte sehr bequem gleichfalls verwerten. Ich denke, ich brauche diese recht einfache Berechnung nicht noch weiter auszuführen.

Nachdem wir früher angedeutet haben, daß wir gewisse Bedenken gegen die Möglichkeit hegen, daß bei der üblichen Sauerstoff-Inhalation das Blut je mit Sauerstoff voll gesättigt wird, werden wir nicht umhin können, diese unsere Bedenken näher zu begründen. $\mathrm{Zu}$ diesem Zwecke müssen wir die drei Arten der Sauerstoff-Applikation, welche in Frage kommen, gesondert besprechen. Mit nur wenigen Worten brauchen wir auf eine Methode der Sauerstoff-Inhalation einzugehen, da sich sofort ergeben wird, daß diese Methode eine durchaus unbrauchbare und aussichtslose ist. Wir denken hier an die Methode, welche noch immer zuweilen angewendet wird, bei welcher der Sauerstoff direkt aus der Bombe, ohne dab ein Reservoir eingeschaltet ist, dem Kranken zugeführt wird. Wird der Hahn der Bombe etwas weiter geöffnet, so daf genügende Mengen von Sauerstoff herausströmen, so entweichen diese unter einem solchen Drucke, daß dabei weder eine Inspiration, noch eine Exspiration möglich ist. Dreht man den Hahn der Bombe wenig auf, so daf der Sauerstoff unter erträglichem Drucke entweicht, so sind das so kleine Mengen, daß man ihnen kaum irgendwelche therapeutische Bedeutung beimessen kann; zum wenigsten kann auf diese Weise nicht so viel Sauerstoff bezogen werden, daß damit die ganze Einatmungsluft bei der Inspiration gedeckt werden kann. Es steht nach wissenschaftlichen Untersuchungen, welche von allen Sachverständigen anerkannt worden sind, fest, daß es im günstigsten Falle möglich ist, Luft unter einem Drucke von $1 / 40$ Atmosphäre einzuatmen. Eine Inspiration dauert zirka zwei Sekunden, welche bei tiefer Einatmung vorübergehend etwas verlängert werden kann. Man kann nun mit Leichtigkeit durch Messung feststellen, dafs bei einem Drucke von $1 / 40$ Atmosphäre in zwei Sekunden nur zirka $11 \mathrm{ccm}$ Sauerstoff aus der Bombe entweichen. Bei dieser Applikationsart des Sauerstoffs stehen also während einer Inspiration bei jenem Drucke nur zirka $11 \mathrm{ccm}$ Sauerstoff zur Verfügung. Wenn man diese kleine Berechnung zugrunde legt, wird man einsehen, daß eine Beimischung von diesen wenigen Kubikzentimetern Sauerstoff zu der Einatmungsluft jedenfalls niemals in der Lage ist, eine Alveolenluft von reinem Sauerstoff zu schaffen, wobei ja erst eine Sauerstoffsättigung des Blutes eintreten könnte, wie sie für unsere Berechnung zugrunde gelegt ist. Wir wollen ganz davon abstrahieren, daß jene $11 \mathrm{ccm}$ Sauerstoff nicht einmal ganz in die Lungen des Kranken eindringen werden, sondern daß davon noch ein gewisser Teil in die freie Atmosphäre entweichen wird. Will man also Sauerstoff-Inhalationen in verständiger Weise ausführen lassen, so muß man stets ein Reservoir einschalten, aus dem der Patient den Sauerstoff einatmet. Daß man ferner leicht gehende In- und Exspirationsventile anwenden mub, um den In- und Exspirationsstrom zu scheiden, versteht sich wohl von selbst.

Es bleiben nun noch zwei Methoden der Sauerstoff-Inhalation übrig, welche wir getrennt besprechen müssen. Die einen bedienen sich hierbei der Gesichtsmasken, die anderen der Mundstücke. Da die Gesichtsmasken anscheinend am meisten Verbreitung gefunden haben, so wollen wir diese Methode zunächst analysieren. Es ist eine bekannte Tatsache, daß alle Gesichtsmasken am Gesichte niemals luftdicht schließen. Daraus ergibt sich von selbst, daß man bei Verwendung derartiger Masken es niemals erreichen kann, reinen Sauerstoff aus dem Reservoir einatmen zu lassen. Bei Gebrauch von Masken wird also der Sauerstoff stets mit gewöhnlicher Luft verdünnt. Wie stark diese Verdünnung des Sauerstoffs ist, das läßt sich zahlenmäßig nicht genau angeben und wird in jedem Falle etwas wechseln. Gering ist diese Zumischung gewöhnlicher Luft zu dem Sauerstoff sicherlich nicht anzuschlagen. Bei dieser Sachlage folgt natürlich, daß es bei Verwendung von Gesichtsmasken nie gelingen kann, dem Kranken reinen Sauer toff einzuführen, sondern nur Gemische von Luft mit Sauerstoff. Ferner wissen wir, daß der fabrikmäßig hergestellte Sauerstoff nicht reiner Sauerstoff ist, sondern nur zu $96 \%$ aus Sauerstoff besteht. Auch der Umstand bedarf der Beachtung, daß die Luft der Lungenalveolen an Sauerstoff stets ärmer ist als die Inspirationsluft. Dieser Verlust beträgt zirka $6 \%$.
Ferner muß berïcksichtigt werden, daß, da die Masken am Gesicht nie vollständig dicht schließen, an eben diesen Stellen der Sauerstoff in die freie Atmosphäre entweichen kann und entweichen wird, ohne überhaupt dem Kranken zugute zu kommen. Wenn wir also diese vier Momente addieren, so dürfte es zweifelhaft sein, ob bei Verwendung von Gesichtsmasken sich je in den Lungenalveolen ein Sauerstoffgemisch von $50 \%$ Sauerstoff vorfinden wird. Selbst wenn wir annehmen wollen, dab bei Gebrauch von Gesichtsmasken in den Alveolen ein Gemisch von $50 \%$ Sauerstoff vorkommt, so würde sich dabei das Blut nicht voll mit Sauerstoff sättigen können, sondern nur zu $93,7 \%$. Jene Zahlen, welche wir früher entwickelt haben, müssen also mindestens noch um $6,3 \%$ reduziert werden, oft wohl noch um mehr. In ähnlicher Weise haben wir natürlich unter diesen Verhältnissen für den im Blute einfach gelösten Sauerstoff eine beachtenswerte Einschränkung zu notierren. Die Menge dieses Sauerstoffs wird bei Gebrauch von Gesichtsmasken infolge der Sauerstoffatmung nicht auf das Fünffache sich steigern können, wie wir früher angenommen haben, sondern nur auf das Zweieinhalbfache. Nun hat man an den Masken für die Sauerstoff-Inhalationen noch eine regulierbare Oeffnung angebracht in der merkwürdigen Furcht, man könne den Kranken sonst zuviel Sauerstoff zuführen. Diese vermeintliche Verbesserung der Sauerstoff-Inhalations-Methode ist eine sichere Verschlechterung und ist auf alle Fälle zu beseitigen. Wenn man einen Patienten mit einer solchen Maske, welche mit Ventilen (selbst mit noch so leicht gehenden) ausgestattet ist, Sauerstoff einatmen läßt, so wird durch diese Methode die Atemmechanik als solche verändert, und zwar in dem Sinne, daß die Atmung vertieft und verlangsamt wird. Wenn sich bei schlecht und oberflächlich atmenden Kranken die Atmung in diesem Sinne verändert, so werden bei der vertieften Atmung Teile der Lunge mit Luft gefüllt und ventiliert, welche vordem nur wenig oder garnicht an der Respiration partizipierten. Ein schlecht und oberflächlich atmender Kranker, der cyanotisch ist, kann, wenn es gelingt, ihn zu bewegen, mit oder ohne diesen Apparat tief und langsam zu atmen, infolge dieser sehr zweckmäßigen Atemgymnastik vorübergehend sein Blut besser mit Sauerstoff versorgen, indem bei der vertieften Atmung mit Aufbietung der auxiliären Atemmuskeln Luft in Teile der Lunge eindringt, welche vorher ungenügend ventiliert worden sind. Auf diese Weise kann unter Umständen eine Cyanose gebessert und vielleicht auch beseitigt werden. Diesen Effekt wird man aber nicht auf eine Wirkung der Sauerstofftherapie beziehen können, sondern genügend mit der veränderten Atemtiefe auf das Konto einer rationellen Atemgymnastik setzen müssen. In zweiter Linie mag vielleicht der erhöhte Sauerstoffgehalt der Einatmungsluft in Betracht kommen; doch ist die Atemgymnastik, die Vertiefung der Atmung sicherlich das bei weitem Wichtigste und die Erhöhung des Sauerstoff-Partiardrucks minder bedeutungsvoll. Eine solche Atemgymnastik läßt sich jedoch nur kurze Zeit unterhalten. Oft bringt man einen dyspnoïschen Kranken überhaupt nicht dazu, in richtiger Weise tief und langsam an diesem Apparate zll atmen, und zwar nie dann, wenn der Kranke bereits tief und langsam respiriert. Da aber ein dyspnoïscher Patient in den schwersten Fällen der Dyspnoë meist bereits tief und langsam atmet, so versagt gerade in diesen schwersten Formen der Atemnot die Atemgymnastik ganz und gar. Allzuviel wird man also von dieser Therapie nicht erwarten dürfen. Ferner wird der Effekt, wo ein solcher überhaupt eingetreten ist, vorüber sein, sobald die Atemgymnastik ausgesetzt wird, oder sehr bald darauf.

Es erübrigt noch, die Methode der Sauerstoffatmung zu besprechen, bei der statt der Masken Mundstücke aus Weichgummi oder Glas Verwendung finden. Die Kranken sollen diese Mundstücke in den Mund nehmen und zwischen Lippen und Zähnen festhalten und dichten. Um dies überhaupt ausführen zu können, dazu gehört ein gewisses Quantum von Kraft. Daß bewußtlose und schwerkranke Patienten mit großer Atemnot diese Mundstücke nie in richtiger Weise gebrauchen können, ergibt sich von selbst. Leichtere Kranke, welche noch genügend Kraft besitzen, werden viel eher mit diesen Mundstücken Sauerstoff einatmen können. Dann wird in der Tat der Sauerstoff unverdünnt aus dem Reservoir eingeatmet und wird auch wirklich in die Lungen eindringen.- Auf diese Weise gelangt in die Lungenalveolen ein Luftgemisch von zirka $90 \%$ Sauerstoff. (Der käufliche Sauerstoff besteht zu $96 \%$ aus Sauerstoff und weitere $6 \%$ gehen verloren, bevor er in die Alveolen kommt.) Auch bei dieser Appli- 
kationsart des Sauerstoffs ist der Umstand zu berücksichtigen, daß dabei die Atmung vertieft und verlangsamt wird, ähnlich wie bei der Sauerstoffatmung mit Gesichtsmasken. Was wir hierüber früher ausgeführt haben, hat auch hier volle Gültigkeit. Wenn wir also von der Wirkung der hierbei eintretenden Aenderung der Atemtiefe abstrahieren, würden auch noch stets $4 \%$ Hämoglobin von Sauerstoff ungesättigt bleiben, da in die Alveolen nur ein Luftgemisch von $90 \%$ Sauerstoff eindringen kann, sodaß jene oben abgeleitete Zahl der Mehraufnahme des Hämoglobins an Sauerstoff eine Reduktion erfahren muß. Auch die Menge des im Blute einfach absorbierten Sauerstoffs wird bei Verwendung der Mundstücke nicht auf das 5 fache sich steigern können, sondern nur auf das $4^{1 / 3}$ fache.

Wenn wir rekapitulieren, so ist es bei benommenen und stark dyspnoïschen Patienten kaum je möglich, eine Sauerstofftherapie in richtiger Weise auszuführen, und auch bei leichteren Kranken wird man den Sauerstoff mit Masken nie unverdünnt bis in die Lungen hineinbringen können, viel eher dagegen mit Mundstücken aus Weichgummi. Daraus ergibt sich, daß es bei Schwerkranken nie gelingen kann, das Blut mit Sauerstoff voll zu sättigen, und auch bei leichteren Dyspnoën wird man das Blut nur bis zu den Graden mit Sauerstoff anreichern, welche wir früher präzisiert haben. Wir stehen daher nicht an zu behaupten, dal wir trotz der sehr interessanten und neuen Untersuchungen von Zuntz und Loewy nicht in die Lage versetzt worden sind, derart neue Gesichtspunkte zu erhalten, daß wir damit nun die Wirksamkeit der Sauerstofftherapie uns physiologisch genügend erklären können. Um nicht mißverstanden zu werden, möchten wir hervorheben, daß wir hier nur solche Krankheiten im Auge haben, bei denen es sich um Kohlensäure-Intoxikationen des Blutes handelt.

Trotz aller Bedenken, welche ich bereits gegen die Möglichkeit einer effektvollen Sauerstoffbehandlung geäußert habe, erscheint mir ein ferneres ganz besondere Berücksichtigung zu verdienen. Wir gelangen hier allerdings auf ein Gebiet der Physiologie, auf welchem trotz sehr zahlreicher Arbeiten noch immer keine volle Einigkeit unter den Physiologen erzielt ist. Während man früher als sicher annahm, daß unter Apıoë ein Zustand $\mathrm{zu}$ verstehen ist, bei dem wegen Sättigung des Blutes mit Sauerstoff nicht geatmet wird, haben wir heute erkannt, daß nicht vor allem der höhere Sauerstoffgehalt des Blutes als vielmehr sein verringerter Kohlensäuregehalt das Maßgebende sei. Andere Physiologen legen das Hauptgewicht bei dem Zustandekommen der Apnoë auf mechanische Momente, welche sie in der Vagusreizung infolge der angestrengten Atmung finden. So viel ist wohl gewiß, daß bei apnoïschen Zuständen oft alle drei Dinge nebeneinander wirken. Es ist daher schwer auszusagen, welches von diesen dreien das wesentlichste ist, ob die Sauerstoffüberladung des Blutes oder seine Kohlensäureverarmung oder aber die Vagusreizullg infolge der angestrengten Atmung. In der menschlichen Pathologie kennen wir sehr gut apnoïsche Zustände, besonders häufig, wenn wir in die Lage kommen, ein Kind wegen einer hochgradigen 'Larynxstenose bei Diphtherie zu tracheotomieren. Sobald das Atmungshindernis beseitigt ist, macht das Kind (selbst wenn es bereits bewuftlos war) einige ungewöhnlich tiefe Inspirationen. Für gewöhnlich tritt dann eine richtige Apnoë ein. Das Kind hört für eine gewisse Zeit auf zu atmen. Auf Unkundige wirkt dieser Zustand direkt beängstigend. Wer dies nicht kennt, glaubt: das Kind atmet nicht, da es tot sei, während es nicht atmet, da es apnoïsch ist. Auch hier hat eine Ueberladung des Blutes mit Sauerstoff stattgefunden. Hand in Hand damit ist eine stärkere Kohlensäureaustreibung aus dem Blute erfolgt, und drittens ist eine ungewöhnliche Reizung der Vagusendigungen in der Lunge bei der forcierten Atmung zustande gekommen. Ganz ähnliche Zustände müßten nach meinem Dafürhalten eintreten, wenn wir bei Einatmung von reinem Sauerstoff eine Sauerstoffsättigung des Blutes erreichen würden, was die meisten Sauerstofftherapeuten annehmen. Sobald das Blut sich mit Sauerstoff sättigt, wird gleichzeitig die Kohlensäure stärker ausgetrieben, und auch die Lungennerven werden infolge der verstärkten Atmung stärker gereizt als bei normaler Atmung. Schlie日lich ist auch noch das Atemzentrum in seiner Erregbarkeit infolge des Sauerstoffmangels und der überanstrengten Atmung bei allen dyspnoïschen Individuen, welche einer 0-Therapie zugänglich sein sollen, stark herabgesetzt. Wir sehen also alle Bedingungen erfüllt, welche für das Zustandekommen der Apnoë von Bedeutung sind, genau wie bei jenem tracheotomierten Kinde. Würde nun in der Tat dabei eine Apnoë eintreten, so würde dieser Umstand an sich eine vermehrte Sauerstoffaufnahme verhindern, eine effektvolle Sauerstoff therapie also illusorisch machen. Da wir andererseits wissen, daß bei Sauerstoffinhalationen solche Apnoëbeim Menschen nie zurBeobachtung kommt, so dürfte der Schluß nicht ungerechtfertigt sein, daß es bei den Sauerstoffinhalationen nicht zu einer Sauerstoffsättigung des Blutes kommt, daß also auch die Kohlensäure nicht besonders intensiv ausgetrieben wird. Dieser Schluf, der ein indirekter ist, dürfte nicht ganz von der Hand zu weisen sein.

Im Anschluf an die früheren Ausführungen, welche wohl geeignet sein düriten, die Unvollkommenheit der Sauerstofftherapie, wie sie bisher in der Klinik und Praxis ausgeführt worden ist, zu beweisen, möchte ich es nicht unterlassen, etwas genauer darauf einzugehen, wie eine zweckmäßige Sauerstofftherapie beschaffen sein müßte. Wie wir gesehen haben, wird bei der bisher gangbaren Sauerstoffinhalation nicht eine reine Sauerstoffbehandlung geübt, sondern wir kombinieren damit eine Atemgymnastik. Es ist daher nicht statthaft, etwaige Erfolge, welche bei Verwendung dieser Art der Sauerstoffinhalation erzielt worden sind, nun ohne weiteres auf die Wirkung des Sauerstoffs zu beziehen. Jedenfalls müssen wir berücksichtigen, daß wir gleichzeitig die Atemmechanik verändert haben. Wir werden also in jedem Falle zu prüfen haben, wie viel bei einer etwaigen Verringerung einer bestehenden Cyanose auf diese Aenderung der Atemmechanik und wie viel auf den Sauerstoff als solchen entfällt. Meine Experimente an Menschen und Kaninchen, sowohl an gesunden als auch an dyspnoïschen, haben ergeben, daß die Aenderung der Atemmechanik das Wesentliche dabei ist, und daß dem Sauerstoff so gut wie keine Bedeutung beizuniessen ist. Wollen wir uns ein eindeutiges Urteil darüber bilden, was die Sauerstofftherapie wirklich zu leisten imstande ist, so müssen wir die Applikation des Sauerstoffs ganz anders einrichten, als dies bisher geschehen ist. Wir haben alles zu vermeiden, was die Atemmechanik verändert. Wie wir uns nach dem Vorschlage von Lazarus ein derartiges eindeutiges Experiment vorstellen, wollen wir nunmehr ausführen. Man müßte sich einen geeigneten, cyanotischen Kranken, etwa einen herzkranken oder cyanotischen Phthisiker oder Bronchitiker auswählen und diesen in einen abgeschlossenen, nicht zu großen Raum, etwa eine pneumatische Glocke, bringen, in welche der Sauerstoff hineingeleitet wird. Bevor man dieses sehr interessante und eventuell entscheidende Experiment anstellt, dürfte es angebracht sein zu überlegen, wie teuer ein solches Experiment sein würde. Ein Mensch gebraucht pro Minute zirka 10 Liter, pro Stunde also 600 Liter Sauerstoff, wenn er reinen Sauerstoff einatmen soll. Wollen wir in unserer pneumatischen Glocke eine genügende Luftventilation unterhalten, so müssen wir pro Kopf und. Stunde 24000 Liter Luft hindurchventilieren. Soll eine gut ventilierte Sauerstoffatmosphäre hergestellt werden, so müssen wir pro Kopf und Stunde 24000 Liter Sauerstoff beschaffen. Wir werden aber natürlich einen etwaigen cyanotischen Kranken nicht allein in der Kammer in reinem Sauerstoff einschließen wollen, sondern werden ihm einen Pfleger oder besser einen Arzt beigeben, sodaß wir pro Stunde 48000 Liter Sauerstoff gebrauchen. Da 1200 Liter Sauerstoff 10 Mark kosten, so würde dieses interessante Experiment sich auf 400 Mark belaufen! Würde man den Versuch auf eine halbe Stunde abkürzen, so wäre der Preis desselben noch immer 200 Mark. Die Sauerstoffbeimengung müßte selbstredend in gleichmäßiger Weise auf die ganze Dauer des Versuches verteilt werden, sodaß alle $1 \frac{1}{2}$ Minuten eine volle Sauerstoffbombe in die Glocke entleert werden müßte. (Dabei dürfte sich die Luft recht bedenklich in unserer Kammer abkühlen.) Es besteht übrigens begründete Hoffnung, daß dieser Versuch über kurz oder lang wirklich ausgeführt werden wird, da eine neue Aktiengesellschaft in Bildung begriffen ist, welche sich die Aufgabe gestellt hat, kleine Zimmer herzustellen, in welche der Sauerstoff hineingeleitet werden soll. Ich hoffe, daf recht bald die Sauerstofftherapie ihre Prüfung abzulegen haben wird. Wünschen wir, dah sie diese Prüfung gut besteht. Eine andere Methode, sich über die Sauerstofftherapie beim Menschen ein untrügliches Urteil zu bilden, gibt es wohl nicht. Diese Applikationsart des Sauerstoffs würde ferner in vollkommenster Weise auch bei Schwerkranken, auch bei Bewuftlosen, Anwendung finden können. Sollte sich nun in der Tat hierbei ergeben, daß der auf diese Weise 
applizierte Sauerstoff imstande ist, die Cyanose zu beseitigen, so wäre damit ein untrüglicher und unbestreitbarer Beweis für die Wirksamkeit der Sauerstofftherapie endgültig erbracht. Nebenbei möchte ich übrigens bemerken, daß es auch bei dieser Methode der Sauerstoffbehandlung nie zu einer vollkommenen Sauerstoffsättigung des Blutes kommen kann, da in die Lungenalveolen nur ein Luftgemisch von $90 \%$ Sauerstoff hineingelangt (vergl. die näheren Ausführungen bei Besprechung der Mundstücke aus Weichgummi). Also auch bei dieser Methode kann es nicht zu einer vollen 0-Sättigung des Blutes kommen, sondern höchstens zu einer Sättigung des Blutes von zirka $96 \%$. Vielleicht ist es nicht überflüssig zu bemerken, daß jener Wert der Sauerstoffsättigung des normalen Blutes von zirka $82 \%$ aus Versuchen von Zuntz und Hagemann hervorgegangen ist, welche an Pferdeblut gewonnen worden sind. Es scheint aber noch auszustehen, ob für den Menschen ähnliche niedere Werte der Sauerstoffsättigung des normalen arteriellen Blutes zutreffen.

Nicht unwesentlich bei Beurteilung der Wirksamkeit der Sauerstoffinhalationen scheint mir ein ferneres Moment zu sein, auf welches bisher meines Wissens nicht hingewiesen ist, obwohl es volle Beachtung verdienen dürfte. Ich denke hier an die Möglichkeit oder Unmöglichkeit einer vollen Sauerstoffsättigung des Blutes bei Einatmung reinen Sauerstoffes. Daf es unter allen Umständen unmöglich ist, selbst bei Einatmung reinen Sauerstoffes, reinen Sauerstoff in die Lungenalveolen hineinzubringen, haben wir bereits auseinandergesetzt. Es erübrigt noch, den Weg weiter zu verfolgen, auf welchem die Alveolenluft in das venöse Lungenblut hineingelangt. Die Lungenalveolen sind bekanntlich von einem platten Alveolarepithel bekleidet, und unter diesem Epithel liegen die Capillarschlingen, in welchen das Lungenblut zirkuliert. Der Sauerstoff der Alveolarluft muß also durch das Epithel der Alveolen und durch die Wandungen der Lungencapillaren hindurch diffundieren. Daf diese Epithelien für die Gasdiffusion ganz besonders günstig konstruiert sind, darüber besteht kein Zweifel. Wenn wir jedoch in Betracht ziehen, daß das Blut in den Lungencapillaren in steter Bewegung sich befindet, welche nicht einmal eine ganz langsame ist, so werden wir uns wohl die Frage vorlegen müssen, ob es in der Tat denkbar ist, daß das venöse Lungenblut in der sehr kurzen Zeit, welche es mit der Alveolenluft in direkten Kontakt kommt (da das Blut ja stetig weiter strömt), je in die Lage kommen kann, sich vollkommen mit Sauerstoff zu sättigen, was wir bei unseren früheren Berechnungen angenommen haben. Dieser Kontakt beträgt nur einen Bruchteil einer Sekunde. Ich persönlich stehe nicht an zu behaupten, daß in dieser kurzen Zeit eine volle Sauerstoffsättigung des Blutes mehr als unwahrscheinlich ist. Bevor nicht ein untrüglicher, experimenteller Beweis dafür erbracht ist, der allerdings die natürlichen Verhältnisse im Tierkörper berücksichtigen muी, bezweifle ich eine derartige volle Sättigung des Blutes mit Sauerstoff. Nach diesen Ausführungen werden wir jetzt zu dem Schlusse kommen müssen, daß alle jene Zahlen der Mehraufnahme von Sauerstoff bei Einatmung reinen Sauerstoffs, welche früher abgeleitet worden sind, sowohl für kranke als auch für gesunde Tierkörper noch viel zu hoch sind und in Wirklichkeit nicht unwesentlich geringer sein dürften. Unsere obigen Berechnungen basieren auf Zahlen, welche nach den ZuntzLoew y schen Formeln berechnet worden sind. Diesen Formeln liegen aber Experimente zugrunde, welche von den Vorgängen im lebenden Tierkörper nicht unwesentlich differieren.

In Kürze möchte ich bei dieser Gelegenheit noch auf den sehr geistreichen Vorschlag Gärtners kritisch eingehen: Sauerstoff intravenös dem kranken Körper zuzuführen. Wir wollen uns die Frage vorlegen: Ist es wirklich möglich, dem cyanotischen Organismus auf diesem Wege mehr Sauerstoff zuzuleiten? Daß man nicht gar zu schnell mit dieser Methode Sauerstoff in das Venenblut hineinleiten darf, das betont schon Gärtner selbst. Injiziert man zu viel Sauerstoff, so gelangt Sauerstoff ungebunden in das rechte Herz; es treten Plätschergeräusche auf, und die weitere Sauerstoffzufuhr auf diesem Wege muß wegen der Gefahren der Luftembolien sistiert werden. Wir wollen voraussetzen, daB die Sauerstoffinfusion richtig dosiert sei. Wird unter diesen Umständen wirklich mehr Sauerstoff vom Tierkörper aufgenommen? Um diese Frage richtig beantworten zu können, müssen wir uns zunächst überlegen: Wie ist unter diesen Umständen das (sonst venöse) Blut der Lungenarterien beschaffen? Während normaliter in den Lungenarterien venöses Blut kreist, fließt bei venöser Sauerstoffzufuhr in den Lungenarterien bereits mehr arterialisiertes Blut. Die natürliche Folge davon dürfte doch wohl die sein, daß nunmehr bei der Atmung weniger Sauerstoff aus der Alveolarluft in das Blut der Lungenarterien übertreten wird. Also die Möglichkeit, auf diesem Wege dem dyspnoïschen Körper mehr Sauerstoff zuzuführen, scheint auch nur in bescheidenen Grenzen vorzuliegen. Auch an dieser Stelle möchte ich ausdrücklich bemerken, daß dies nur für die Kohlensäureintoxikationen des Tierkörpers zutrifft. Für die Kohlenoxydvergiftungen liegen die Verhältnisse sehr wesentlich anders.

Nach allen diesen Ausstellungen, welche ich gegen die Wirksamkeit der Sauerstofftherapie bei Kohlensäurevergiftungen aller Art motiviert habe, kann ich nicht umhin, meine Bedenken gegen die Wirkung der Sauerstoffbehandlung aufrecht $\mathrm{zu}$ erhalten, trotz der neuen und wichtigen Untersuchungen von Zuntz und Loewy. Auch meine Beobachtungen am Krankenbette haben mich bisher nicht davon überzeugt, dail meine Kritik der Sauerstofftherapie eine $\mathrm{zu}$ strenge gewesen ist. Es dürfte vielleicht angebracht sein, an dieser Stelle eine typische und beherzigenswerte Beobachtung wiederzugeben, welche ich vor einiger Zeit erlebt habe. Es kam ein kleines Mädchen zu uns in die Poliklinik mit einem angeborenen Herzfehler (Pulmonalfehler). Seine Cyanose war eine außerordentlich ausgeprägte. Man konnte fast von einer Blausucht sprechen. Das Kind atmete schnell und oberflächlich. Ich benutzte die günstige Gelegenheit, um den Effekt der Sauerstoffinhalation wieder einmal zu prüfen. Ich lieh also Sauerstoff aus einem Spirometer mit einem Weichgummimundstücke einatmen. Nachdem das Kind die erste Aengstlichkeit überwunden hatte, respirierte es ganz leidlich an dem Apparate. Schon nach wenigen Atemzïgen war die Cyanose so deutlich gebessert, daß wohl niemand diese Tatsache bestreiten konnte. Nach Aussetzen der Sauerstoffinhalation wurde das Kind sehr bald wieder so cyanotisch wie zuvor. Darauf lieb ich aus dem Spirometer statt Sauerstoff gewöhnliche Luft inspirieren, und sofort besserte sich die Cyanose ebenso augenfällig wie bei der Sauerstoffinhalation. Hätten wir uns mit dem ersten Versuche begnügt, so hätten auch wir einen bemerkenswerten Beitrag für die außerordentliche Wirksamkeit der Sauerstofftherapie erbracht.

Nach all diesen Momenten kann ich bisher meine Bedenken gegen die Wirksamkeit der Sauerstoffinhalationen bei Kohlensäurevergiftungen des Tierkörpers nicht fallen lassen. 